\title{
Exponential and Separation of Variables Exact Solutions to the Linear, Delayed, Unidirectional Wave Equation
}

\author{
Ronald E. Mickens' ${ }^{1}$ and 'Kale Oyedeji ${ }^{2, *}$ \\ ${ }^{1}$ Department of Physics, Clark Atlanta University, \\ Atlanta, GA 30314, USA

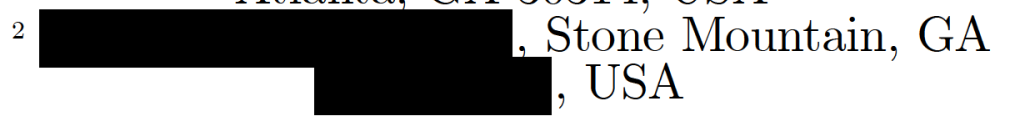

\begin{abstract}
We calculate sone exact solutions to the linear,delayed, unidirectional wave equation using the method of separation-of-variables and an exponential type ansatz. Some important features of these solutions are indicated and their stabilities are examined. An order-four Taylor series is determined for the exponential-type solutions where the expansion is in terms of the delay parameter. Some brief comments on the delayed diffusion and other physical based equations are also given.
\end{abstract}

Key words: Delayed differential equations, exact solutions, Lambert-W function, Unidirectional wave equation

AMS Classifications: $35 \mathrm{C}) 5,33 \mathrm{~F} 0 \mathrm{~S}, 35 \mathrm{~F} 99$

* Corresponding author: kaleoyedeji@comcast.net

Emails: rmickens@cau.edu, kaleoyedeji@comcast.net 


\section{Introduction}

Both ordinary [1] and partial differential equations [4,5] provide models for a diverse set of phenomena in the natural and engineering sciences. However, few of these equations have general solutions expressible in terms of elementary functions $[1,6]$. For many such equations special solutions can be determined by applying the method of separation-of-variables (SOV) [6]. An issue of importance, especially for scientific applications, is the stability of a given solution. Often the desired stable solution either does not exist or is difficult to calculate; see $[8]$ as an example of such problems.

The main purpose of this short note is to construct some exact solutions to the linear, delayed, unidirectional wave equation having constant coefficients. It is given by the following expressions.

$$
U_{t}(t+\tau, x)+U_{x}(t, x)=0,
$$

where

$$
U_{t}=\frac{\partial U}{\partial t}, \quad U_{x}=\frac{\partial U}{\partial x}
$$

and $\tau$ is the delay parameter. (It should be indicated that our search of the electronic based research literature did not provide any references for this delayed differential equation.) This is perhaps the most elementary of the partial differential delayed equations and should allow us to explicitly calculate some particular solutions. This is done using two techniques. The first is the method of separation-of-variables. The second procedure is based on the assumption that the solution has an exponential structure.

The paper is planned as follows. Section 2 solves for a separation-of-variable solution. We find that the obtained solution involves the Lambart-W function [2]. Exponential type solutions are constructed in section 3. Finally, in section 4 , we give a brief summary of the results and provide a short discussion on the physical basis for delay differential equations.

\section{Separation-of-Variable (SOV) Solutions}

First, observe that for $\tau=0$, Eq.(1.1) becomes

$$
U_{t}(x, t)+U_{x}(x, t)=0,
$$

and this equation has the general solution

$$
U(x, t)=f(x-t)
$$

an important particular solution is

$$
U_{p}(x, t, k)=e^{i k(x-t)}
$$

If we now "sum" over $\mathrm{k}$, then we have

$$
U(x, t\}=\int_{-\infty}^{\infty} \Phi(k) U_{p}(x, t, k) d k,
$$


and require

$$
\Phi(-k)=\Phi(k), \quad \int_{-\infty}^{\infty}|\Phi(k)| d k<\infty,
$$

then $\mathrm{U}(\mathrm{x}, \mathrm{t})$ depends only on $\mathrm{U}(\mathrm{x}-\mathrm{t})$ and is bounded.

Second, using the SOV method, a special solution to

$$
U_{t}(x, t+\tau)+U_{x}(x, t)=0,
$$

takes the form

$$
U(x, t)=f(x) g(t)
$$

Using

$$
U x, t+\tau)=f(x) g(t+\tau),
$$

and

$$
U_{x}(x, t)=f^{\prime}(x) g(t), \quad U_{t}(x, t+\tau)=f(x) g^{\prime}(t+\tau),
$$

where the prime denotes taking the appropriate derivative, Eq. (2.6) becomes

$$
f(k) g^{\prime}(t+\tau)+f^{\prime}(k) g(t)=0 .
$$

Dividing each term by $\mathrm{f}(\mathrm{x}) \mathrm{g}(\mathrm{t})$ gives

$$
\frac{g^{\prime}(t+\tau)}{g(t)}+\frac{f^{\prime}(k)}{f(k)}=0,
$$

and this expression implies that (for the separation constant $k$ )

$$
\begin{aligned}
& \frac{d g(t+\tau)}{d t}=k f(x), \\
& \frac{d f(x)}{d x}=-k f(x) .
\end{aligned}
$$

The solution to the last equation is

$$
f(x)=c_{1}(k) e^{-k x},
$$

where $c_{1}(k)$ is an arbitrary function of $\mathrm{k}$.

If we now assume that Eq. (2.12) has a solution of the form $[1,2\}$

$$
g(t)=e^{r t}
$$

then its substitution into Eq.(2.6) gives, after some simplifications, the result is

$$
r e^{r \tau}=k
$$

This latter equation can be rewritten as

$$
(r \tau) e^{r \tau}=k \tau .
$$


However, the Lambert-W function is defined by the relation [2]

$$
W(z) e^{W(z)}=z,
$$

and, consequently, a comparison of Eq.(17) and Eq.(2.18) gives

$$
r=\frac{1}{\tau} W(k \tau),
$$

and

$$
g(t, k, \tau)=e^{\frac{1}{\tau} W(k \tau) t} .
$$

Therefore

$$
U(x, t, k, \tau)=e^{-k x} e^{\frac{1}{\tau} W(k \tau) t} .
$$

Since the delayed PDE is linear, then a "general" solution, based on the SOV method, takes the form

$$
U(x, t, \tau)=\int A(k) e^{-k x} e^{\frac{1}{\tau} W(k \tau) t} d k,
$$

where $\mathrm{A}(\mathrm{k})$ is such that the integral is defined.

Using the fact that the Taylor series around $\mathrm{z}=0$ is

$$
W(z)=z-z^{2}+\frac{3}{2} z^{3}+\ldots
$$

it follows that

$$
\operatorname{Lim}_{\tau \rightarrow 0} \frac{W(k \tau)}{\tau}=k,
$$

and, therefore,

$$
U(x, t, 0)=\int A(k) e^{-k(x-t)} d k=F(x-t),
$$

as expected from Eq. (2.6).

\section{Exponential Solutions}

The delayed PDE

$$
U_{t}(x, t+\tau)+U_{x}(x, t)=0
$$

has exponential solutions of the form

$$
U(x, t)=e^{(a x+b t)},
$$

where the parameters $(a, b)$ are to be determined. In general, a functional relationship is expected between them.

A direct calculation gives

$$
U(x, t+\tau)=e^{b \tau} U(x, t),
$$




$$
\begin{gathered}
U_{t}(x, t+\tau)=b e^{b \tau} U(x, t), \\
U_{x}(x, t)=a U(x, t) .
\end{gathered}
$$

Substituting these relations into Eq. (3.1) and simplifying the resulting expression gives a solvability condition, namely

$$
a=-b e^{b \tau} .
$$

We will use this to define $A$ in terms of $b$ and this is much easier to deal with than solving for $\mathrm{b}$ as a function of $\mathrm{A}$; the latter possibility introduces the Lambert-W function [2].

With this result, we have the exponential type solution

$$
U(x, t, b, \tau)=\exp \left[\left(-b e^{b \tau}\right) x+b t\right]
$$

Therefore, a general solution takes the form

$$
U(x, t, \tau)=\int A(b) U(x, t, b, \tau) d b,
$$

where $\mathrm{A}(\mathrm{b})$ is a function such that the integral exists.

Again, observe that from Eq.(3.)

$$
\operatorname{Lim}_{\tau \rightarrow 0} U(x, t, b, \tau)=e^{-b(x-t)},
$$

and from Eq. (3.6)

$$
\operatorname{Lim}_{\tau \rightarrow 0} U(x, t, \tau)=G(x-t)
$$

To provide some possible insights into the small $\tau$ behavior of $\mathrm{U}(\mathrm{x}, \mathrm{t}, \mathrm{b}, \tau)$, we now calculate the first several terms of the Taylor series expansion around $\tau=0$, i.e.,

$$
U(x, t, b, \tau)=U x, t, b, 0)+\tau \frac{\partial U(x, t, b, 0)}{\partial \tau}+\frac{1}{2} \tau^{2} \frac{\partial^{2} U(x, t, b, 0)}{\partial \tau^{2}}+\ldots .
$$

While the derivatives can be directly calculated from Eq.(3.5), their actual computation is algebraically intense. The completion of this task gives the following formal expression for the Taylor series, up to terms of order $\tau^{4}$ :

$U(x, t, b, \tau)=e^{-b(x-t)}\left\{1+\tau(b B)\left[\begin{array}{c}1+\frac{1}{2} b(1+B) \tau+\frac{1}{6} b^{2}\left(1+3 B+B^{2}\right) \tau^{2}+ \\ \frac{1}{24} b^{3}\left(1+7 B+6 B^{2}+B^{3}\right) \tau^{3}+O\left(\tau^{4}\right)\end{array}\right]\right\}$ 
where

$$
B=(-b x)
$$

An examination of Eq. (3.12) implies that the full formal Taylor series has the following structure

$U(x, t, b, \tau)=e^{-b(x-t)}\left\{1-\left(b^{2} x\right) \tau\left[\begin{array}{c}1+b P_{n}(x) \tau+b^{2} P_{2}(x) \tau^{2}+b^{3} P_{3}(x) \tau^{3} \\ +\ldots+b^{n} P_{n}(x) \tau^{n}+\ldots\end{array}\right]\right\}$

where $P_{n}(x)$ is an $\mathrm{n}$-th degree polynomial in $\mathrm{x}$. A naive, but almost certain to be true conjecture, is that this expansion is not convergent.

\section{Discussion}

In this preliminary set of calculations related to the linear, delayed, unidirectional wave equation, we have constructed two classes of solutions. The first was based on the method of separation-of-variables (SOV) while the second writes the down an a priori expressed exponential type ansatz. Note that the two methods are not independent of each other since the exponential form of the solution is a special case of the SOV technique.

One likely conclusion, obtained by expanding the exponential type solution in a formal Taylor series, is that this series may not be, in the delay $\tau$, convergent. A related issue is whether the solutions to this equation, Eq.(1.1), are stable. The results of Jordan et al. [3] give reasons to believe that most, if not all are unstable. This problem clearly needs to be investigated in more detail.

If Eq.(1.1) is expanded to first order in $\tau$, then the following second-order PDE is obtained

$$
U_{t}(x, t)+\tau U_{t t}(x, t)+U_{x}(x, t)=0 .
$$

Such procedures have become standard in many areas of the physical and engineering sciences $[3,7]$, with the main reasoning being that PDE's, such as Eq.(4.1), may be easily solved by a variety of already known techniques, while delay PDE's are much more difficult to both analyze and solve (generally by numerical methods). An interesting and important problem would be to study the properties of Eq.(1.1) and compare them with our SOV and exponential-type solutions.

Finally, it should be noted that the Lambert-W function, defined in Eq. (2.18), has an infinite number of branches, and hence solutions. However, this was not explicitly indicated in the calculations presented in Section 2

\section{References}

[1] R. Bellman, K.L. Cooke, Differential-Difference Equations, Academic Press, New York, 1953

[2] R.M. Corless, G.H. Gonnet, D.E.G. Hare, D.J. Jeffrey, D.E. Knuth, On the Lambert $W$ function, Advances in Coputational Mathematics 5(1996) 329359. 
[3] P.N. Jordan, W Dai, R.E. Mickens, A note on the delayed heat equation: Instability with respect to initial data, Mechanics research Communications 35(2008) 414-420.

[4] Y. Kuang, Delay Differential Equations with Application in Population Dynamics, Academic Press, Boston, 1993.

[5] A.D. Polyanin, V.F. Zaitsev, Handbook of Nonlinear Partial Differential Equations, Chapman and Hall/CRC, Boca Raton, 2012.

[6] A.D. Polyanin, A.I. Zhurov, New generalized and functional separable solutions to non-linear delay reaction-diffusion equations, International Journal of Non-Linear Mechanics, 59 (2914) 16-32.

[7] A.V. Vyazmin and V.G. Sorokin, Exact solutions to nonlinear delay differential equations of hypolic type. IOP Conference Proceedings: Journal of Physics 788(2017) 012037. 